\title{
MIGRATIONS ET DEVELOPPEMENT EN AFRIQUE : QUELQUES ASPECTS METHODOLOGIQUES
}

\author{
par \\ LUTUTALA Mumpasi \\ Département de Démographie \\ Université de Kinshasa
}

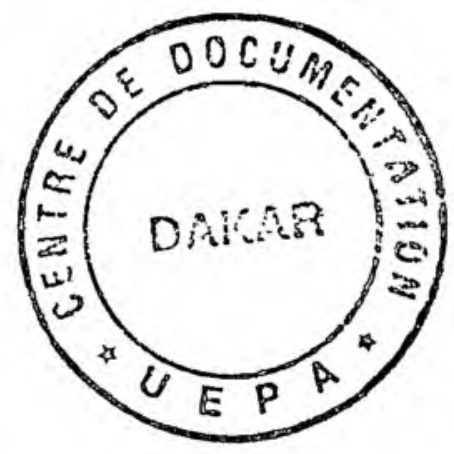


On dispose présentement de plusieurs travaux sur les aspects méthodologiques des migrations africaines, notamment sur la qualité des sources de données, les limites des méthodes classiques d'analyse démographique, les limites du concept même de migration, mais moins sur le rôle de la migration dans le processus de développement. Ces travaux ne sont pas seulement l'oeuvre des auteurs individuels, des organisations comme la Communauté Economique pour 1'Afrique (CEA) ou les Nations Unies (Division de Population) ont aussi publié des études sur ces aspects. La CEA, par exemple, a consacré trois numéros - $\mathrm{n}^{\circ} 14,15$ et 17 - du BIS (Bulletin d'Information Statistique) aux migrations.

De tous ces travaux, trois synthèses-critiques relativement récentes (Bilsborrow, 1979; Gregory et Piché, 1985 et Simmons, 1985) restent des références obligées pour toute nouvelle réflexion méthodologique sur les migrations africaines. D'autres études sont également à retenir pour certains aspects particuliers des migrations, notamment celui de Gregory (1989) sur les questions méthodologiques de l'étude de l'insertion des migrants en ville.

Le travail de Bilsborrow (1979) avait été préparé dans le cadre d'une réflexion conduite par les Nations Unies sur la modelisation de la dialectique population-développement. L'auteur arrivait à la conclusion que les études réalisées par les économistes n'avaient pas permis (jusqu'en 1979, mais les choses auraient-elles changé depuis ?) une meilleure connaissance du phénomène migratoire (p.79). Pour Bilsborrow, ceci serait dû au fait que les "causes" des migrations sont étudiées indépendamment des conséquences ; ce qui permet certes de savoir si une politique migratoire est souhaitable, mais non le type de politique à préconiser. Mais il y a aussi le fait que les recherches sont basées sur des données individuelles, inadéquates, collectées généralement pour d'autres fins et souvent dans les milieux urbains de destination.

Dans leur étude, Grégory et Piché (1985) examinent les objectifs, les méthodologies et les résultats des enquêtes sur les migrations réalisées dans neuf pays africains au courant des années '60 et '70. Ces auteurs arrivent à deux conclusions très importantes pour l'étude des migrations africaines :

1) il est illusoire de tracer un bilan strictement (c'est nous qui soulignons !) méthodologique des enquêtes, car tout dépend de la finalité de la recherche (p.344). Une donnée statistique, une méthode de collecte ou d'analyse n'a d'utilité que par rapport à une problématique. Et comme le dit ailleurs Grégory (1979:161), "pour s'interroger sur les méthodes, il est d'abord nécessaire de bien définir l'objet d'étude".

2) la somme impressionnante des connaissances sur les migrations africaines ne nous renseigne que très peu sur les dimensions sociales et historiques du phénomène, ou si l'on veut sur ses interrelations avec le processus de développement. 
Pour sa part, Simmons (1985) arrivait quasiment à la même conclusion : les enquêtes sur les migrations ne permettent pas encore une meilleure observation du phénomène migratoire dans tous ses aspects. Elles se sont plus focalisées sur la migration rurale-urbaine, la migration durée-de-vie interrégionale, mais beaucoup moins sur le processus socio-économique qui est rattaché au phénomène. Quatre raisons expliqueraient cela : les conséquences socio-politiques du phénomène, les considérations théoriques sur le rôle des migrations dans le processus de développement, les contraintes liées aux coûts des enquêtes sur la migration, et la pesanteur des méthodologies par trop conventionnelles. Par ailleurs, ces enquêtes n'ont pas été réalisées conjointement avec un effort de re-conceptualisation du phénomène. Comment alors, s'interroge Simmons, permettre aux enquêtes de mieux observer le phénomène dans tous ses aspects ? Comment réussir l'étude des migrations en rapport avec le processus de développement ? La conclusion à laquelle il arrive est une véritable interpellation : l'étude des migrations exige d'importantes innovations méthodologiques, mais celles-ci doivent elles-mêmes faire l'objet de recherches.

Il ressort de ce qui précède qu'une meilleure connaissance des interrelations de la migration avec le processus de développement reste handicapée par des écueils méthodologiques de taille. Ce qui ne permet pas, en conséquence, de mieux prendre en compte ce phénomène dans le processus de planification du développement et/ou de formuler une politique migratoire réaliste. Le but de ce papier est d'examiner quelques uns de ces écueils qui nous paraissent les plus importants.

Pour ce faire, et suivant en cela les préoccupations des auteurs précités, une question concernant le rôle des migrations dans le processus de développement est à se poser préalablement. Une fois cette conceptualisation réussie, nous verrons alors quelles sont les variables que toute source de données doit saisir pour permettre une telle étude. Nous examinerons ensuite le problème lié aux données et aux méthodes d'analyse.

\section{Migrations et développement : un cadre conceptuel}

Le rôle et la place des migrations dans le processus de développement sont généralement examinés au niveau micro et macro. En fait ces deux approches devraient être utilisées simultanément (Piché, 1989:202).

Au niveau micro, les économistes néo-classiques considèrent le migrant comme un individu rationnel dont la décision de migrer découle d'une évaluation négative de sa situation actuelle et positive des revenus espérés au lieu de destination, et cela compte tenu du coût de la migration. C'est le modèle "coûts-bénéfice". Les flux migratoires dans leur ensemble vont permettre, au niveau macro, le développement ; car les migrations vont consister en un transfert de la force de travail des milieux ruraux où elle est surabondante et moins rentable (le capital y étant rare) vers les "pôles de développement" (généralement les milieux capitalistes urbanisés) où elle est insuffisante, plus recherchée et plus productive, ce qui permet de 
générer la croissance économique. Celle-ci va permettre un ajustement sectoriel et régional gràce à des mécanismes comme le renvoi des fonds au milieu d'origine, le réinvestissement du surplus généré par la production,la répartition des fruits du développement, etc.

Nous n'allons pas, dans ce papier, valider cette thèse optimiste néo-classique. Nous nous limiterons à mentionner quelques paradoxes auxquels la migration conduit aussi bien dans les milieux ruraux d'origine que dans les milieux urbains de destination, pour ne considérer que les migrations rurales-urbaines qui préoccupent essentiellement les économistes. Pour ce faire, nous nous servirons du schéma 1 de la page suivante, ce que nous empruntons à Bisborrow (1976). Il faut tout de suite dire que ce schéma reste à améliorer notamment en y insérant l'interaction entre la migration et les autres composantes de l'accroissement démographique, à savoir la fécondité et la mortalité. Mais il faut aussi considérer la dynamique que la migration instaure entre les milieux d'origine et de destination, qui n'y apparaît pas clairement.

On peut résumer ces processus en disant qu'en transférant la force de travail dans les milieux de destination, la migration y crée des besoins en logements, écoles, hôpitaux, transports, emplois, etc. que "1'output" qu'elle génère n'est pas capable de satisfaire. Il s'ensuit évidemment un sousdéveloppement urbain qui a déjà fait l'objet de plusieurs travaux. Mentionnons ici celui de Deblé et Hugon (1982). Le titre de leur ouvrage, "Vivre et survivre dans les villes africaines" est suffisamment éloquent, nous semblet-il, pour qu'on s'y appesantisse ! Il est dès lors difficile de concevoir que la ville, ou les migrants qui y vont, ou l'industrie qui les y utilise, soient un moteur du développement rural, via les fonds rapatriés par les migrants, les investissements qu'ils $y$ réalisent ou que d'autres (Etat ou autres investisseurs) y réalisent. Et quand bien même il en serait ainsi, il a été démontré ailleurs que les fonds rapatriés par les migrants ne peuvent être vus comme des investissements créateurs d'emploi ou augmentant la productivité, mais bien des fonds pour des dépenses ostentatoires (Locoh, 1985:359). Le milieu rural continuera donc à souffrir de cette ponction de sa force de travail qu'est la migration.

Les considérations que nous venons d'émettre ne sont pas de simpies spéculations théoriques. En effet, les migrations et la distribution spatiale de la population qu'elles occasionnent sont considérées par la quasi-totalité des gouvernements africains comme un des problèmes démographiques les plus sérieux qu'ils connaissent (Nations-Unies, 1983 et 1986:80). Elles seraient même "à l'origine d'un plus grand nombre de problèmes de développement que ne l'est la croissance démographique elle-même" (Nations Unies, 1985:30). Ainsi en est-il, par exemple, des conséquences des migrations sur "l'explosion urbaine" et la dévitalisation des milieux ruraux. Pour les décideurs politiques, il s'agit là, nous le verrons, de problèmes "chauds" qui défient la crédibilité des systèmes politico-économiques et la stabilité des Partis au pouvoir.

On aura remarqué, de ce qui précède, qu'il semble bien exister une relation dialectique entre les considérations néo-classiques des migrations et les 


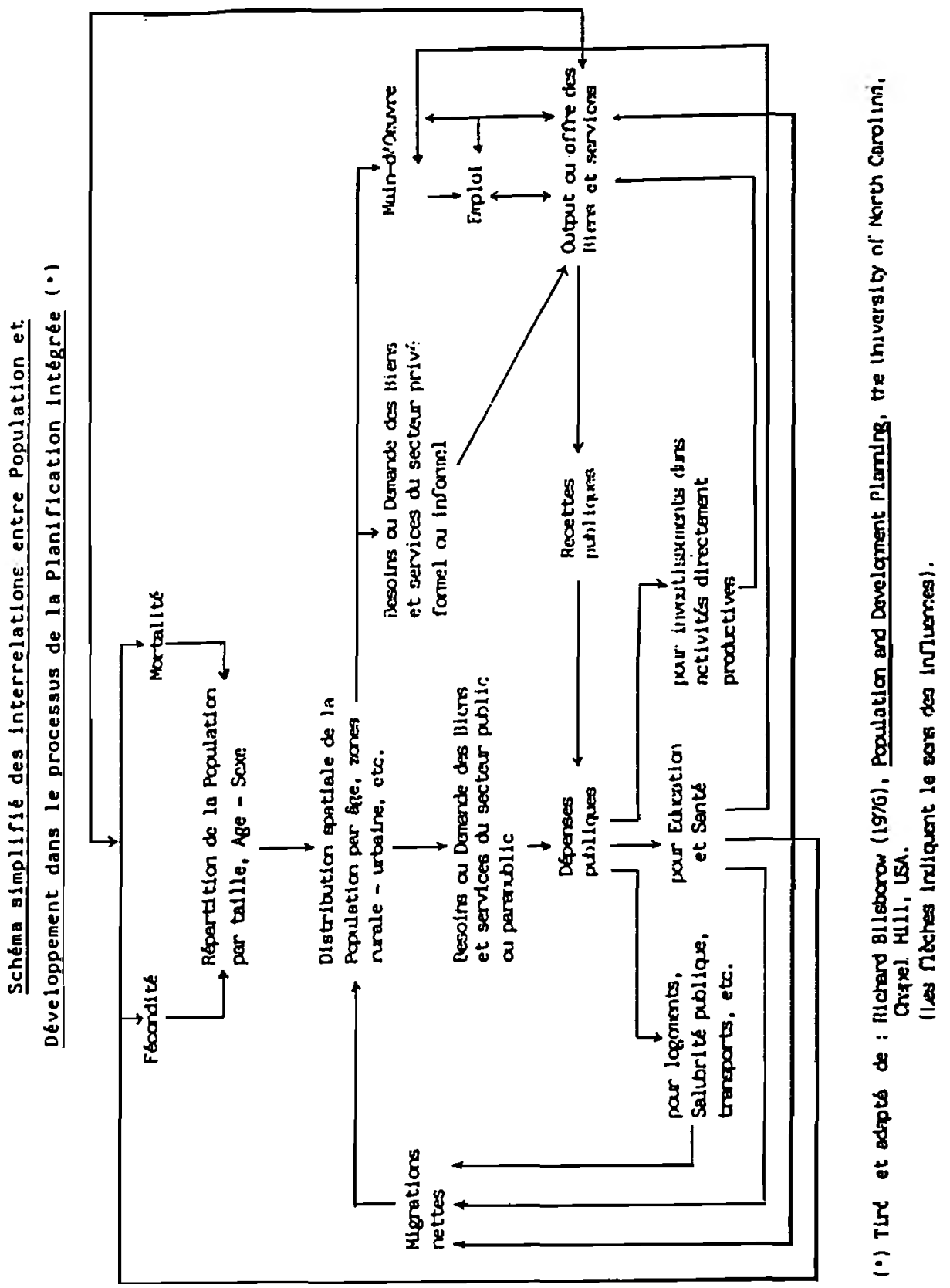


conséquences telles que vécues par les ménages, les familles et les individus. Ce qui rend indispensable une réinterprétation du phénomène. En effet, les rapports sociaux de production et de reproduction des pays africains sont tels que les individus, les ménages et les familles sont obligés de développer des stratégies de survie, dont les migrations. Celles-ci assurent à la famille ou au ménage une place au sein des rapports sociaux du milieu de destination, ce qui leur permet d'y réaliser la productionreproduction extra-locale, et vice-versa. Un aspect important à étudier serait donc le processus et le succès ou l'échec de l'insertion des migrants dans le nouveau milieu.

L'existence des membres extra-locaux instaure une intéraction entre eux et les membres locaux, dont la nature et l'intensité dépendront de leur plus ou moins forte insertion au milieu de destination, laquelle dépend à son tour des rapports sociaux au milieu d'origine, du sexe du migrant, et de sa classe sociale. En influant sur les rapports sociaux respectifs, cette intéraction dynamise la production-reproduction, le comportement démographique et le statut social (le niveau de développement si l'on veut) des membres familiaux, locaux et extra-locaux.

Le schéma 2 ci-dessous tente de résumer ces relations...

Schéma conceptuel sur miqrations et développerment -analyse au niveau micro-

$\mathbf{i , t}$

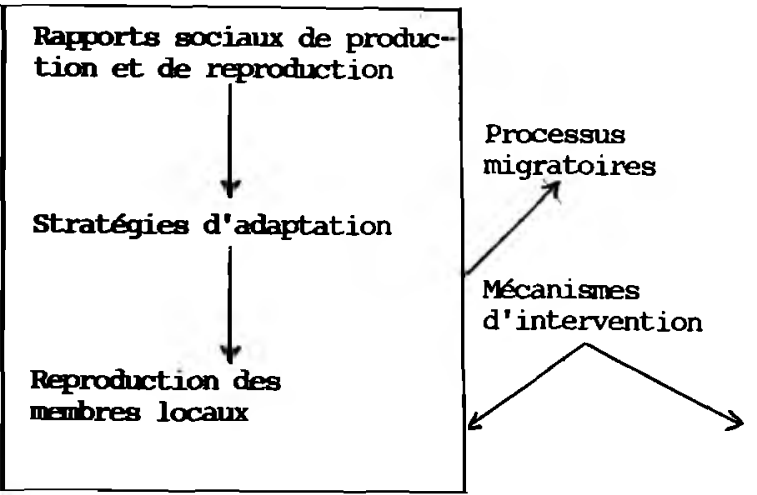

$j, t+n$

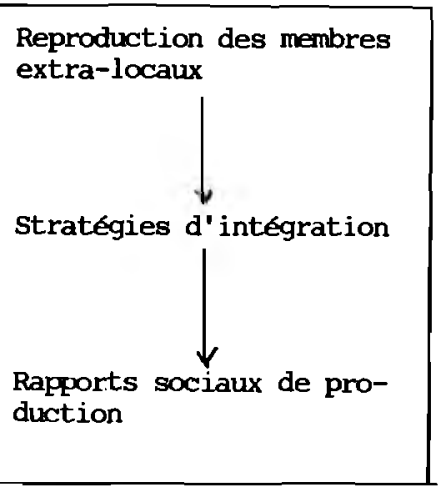

source: Lututala $(1987: 32)$.

\section{Les variables à saisir et à analyser}

Le cadre conceptuel qui vient d'être brossé montre que la migration se situe au centre du processus de développement ou de sous-développement. La migration et le développement entretiennent donc une relation dialectique : l'un et l'autre est à la fois "cause" et conséquence. Et l'un et l'autre sera tantôt une variable expliquée, tantôt explicative. Les données à collecter doivent par conséquent porter sur les deux concepts.

Au niveau macro-économique, l'étude des "causes" des migrations nécessitera des données sur le couple production-développement d'une part et d'autre part sur les migrations nettes (soldes migratoires nets et intentions des 
migrants de demeurer ou non dans les nouveaux lieux de résidence). L'objectif visé ici sera de montrer dans quelle mesure les structures de production sont responsables de l'intensité et des formes de migration qu'on observe et vice-versa. Un tel objectif va au-delà de la vision déterministe néo-classique, où, rappelons-le, l'effet des structures de production sur les migrations est considéré d'avance comme positif et salutaire. Une gamme trèș variée de variablea peut être utilisée pour mesurer le développement (et son corrolaire la production) : le PNB, l'évolution des structures d'emploi, le développement inégal des régions, le caractère extraverti de l'économie, les termes d'échange de l'agriculteur-paysan, l'expropriation foncière des ruraux, la stratification sociale, etc. (Piché, 1989:206 ; Simmons, 1985:236). D'autres variables "communautaires" sont suggérées par Bilsborrow (1984) : possibilités d'emploi, niveaux de salaires, systèmes fonciers, liens de parenté, réseaux de communication et de transport, accès aux services publics et communautaires, structures politiques et économiques, facteurs climatiques, etc.

Par ailleurs, l'impact de la migration sera recherché sur la distribution spatiale de la population, la production et les dépenses publiques (voir schéma 1). Pour la distribution spatiale, il sera nécessaire de disposer des données sur les soldes migratoires nets ainsi que l'intention des migrants de demeurer ou non à leur nouveau lieu de résidence. L'impact sur les taux d'activité économique (main-d'oeuvre, emploi) nécessitera quant à lui des données sur les caractéristiques des migrants selon 1'âge, le sexe, l'état matrimonial, le type de migrants (migrants primaires, secondaires dépendants ou secondaires indépendants), le rang dans la fratrie, la structure d'accueil, etc. L'impact de la migration sur la production (1'output) exigera des données sur d'autres caractéristiques des migrants : lieux d'origine, scolarité, profession, ethnie d'appartenance, etc. Enfin, l'impact sur les dépenses publiques exigera des données sur la mobilité géographique et professionnelle des migrants, leur localisation résidentielle, la structure de leurs dépensẹs, leurs caractéristiques socio-démographiques.

Si l'analyse de la migration au niveau macro doit viser à montrer la responsabilité des structures de production sur le phénomène, c'est, pensơns-nous, la façon avec laquelle les ménages et les individus composent avec ces structures de production, via la migration et réussissert leur stratégie qu'il faudrait viser au niveau micro. Dans ce sens, la migration n'est pas seulement un phénomène passif par rapport aux structures de production ; au contraire, elle peut aussi les influencer, positivement ou négativement. Pour le démontrer, il faudrait disposer de données sur les mécanismes d'insertion des migrants dans les nouveaux milieux ; leurs relations avec les communautés d'origine ; leurs caractéristiques avant et après la migration ; leurs opinions sur la migration ; leurs biographies migratoire, professionnelle et familiale; etc.

\section{Les écueils méthodologiques}

La problématique migration-développement étant posée, et les variables à 
saisir pour l'analyser identifiées, venons-en maintenant à la question de savoir s'il est possible, dans l'état actuel des choses, de procéder à une telle étude avec satisfaction. Nous examinerons pour ce faire l'état actuel des statistiques et des méthodes d'analyse.

\subsection{L'absence des données}

\subsubsection{Les données socio-économiques}

Le processus de planification et de prise de décision socio-économique en Afrique reste fortement handicapé, on le sait, par un manque total de données (absence absolue) ou de données fiables, récentes et pertinentes (absence relative). L'étude de la migration souffre donc directement de ce manque de données socio-économiques.

Plusieurs raisons peuvent être évoquées pour expliquer cette situation. La plus importante pour nous est le rôle et la place marginaux accordés à la recherche scientifique en général, démographique en particulier dans le processus de planification (si planification il y a !) et de prise de décision. Car la valorisation de la recherche occasionnerait ipso facto la collecte, le traitement et le stockage de données en vue de poser les questions de développement et d'y répondre.

La valorisation de la recherche et des données scientifiques n'est cependant possible que si (1) les liens entre la recherche et le développement sont bien établis et (2) le développement est recherché pour la population, pour l'amélioration de ses conditions de vie et n'est pas synonyme d'exploitation, d'assujettissement, etc. Les pays africains étant encore loin de cet idéal (c'est là notre postulat), un travail de sensibilisation mérite d'être mené à ce niveau.

Le peu de données dont on dispose sur la situation socio-économique sont généralement des données macro. Peu d'efforts sont fournis pour recueillir les données au niveau des communautés, telles que prévues par Bilsborrow. Par ailleurs, la qualité des données micro-économiques reste encore à améliorer. Les données sur la profession, par exemple, sont incapables de montrer toute la complexité qui existe entre l'individu et son marché du travail, et donc de situer l'individu dans la stratification sociale (cfr Gregory, 1989:167-168).

\subsubsection{Les données sur la migration}

Ce qui vient d'être dit des données scientifiques en général reste vrai pour ce qui est des données démographiques. Ceci dit, cette section va tenter de procéder à une "critique. interne" des statistiques sur les migrations qui existent et qui peuvent permettre l'étude de la dialectique migrationdéveloppement.

Trois phases semblent caractériser l'état des statistiques sur les migrations. La première phase, celle de l'époque coloniale, est caractérisée par un 
intérêt très soutenu et une masse importante des données sur les "migrations". L'intérêt de collecter les statistiques sur le phénomène était aussi grand, sinon plus, que celui porté sur les problèmes et les données concernant l'infécondité. C'est que le souci du colonisateur de disposer d'une main-d'oeuvre pour réussir l'exploitation coloniale a fait de la population une variable-clé. Au Zaïre, alors Congo-Belge, le souci de connaître les effectifs de main-d'oeuvre mobilisée (les "migrants" recrutés) et mobilisable a même été à la base des opérations de collecte des données appelées "coups de sonde".

Les statistiques coloniales sur les migrations africaines souffrent cependant de plusieurs lacunes. D'abord, elles portent rarement sur l'ensemble du pays concerné. Bien au contraire,' il s'agit souvent de données portant sur quelques villages, et surtout les centres urbains, où les statistiques des "arrivées" étaient bien tenues. Il faut signaler en deuxième lieu que ces statistiques portent sur une conceptualisation des migrations trop étroite et inadaptée aux réalités africaines. En effet, deux questions étaient généralement posées pour saisir les migrations : le lieu de naissance et l'appartenance ethnique. Ce qui conduisait à considérer comme migrant toute personne qui se trouvait en dehors de son lieu de naissance ou du lieu de localisation de son ethnie. Dans certains pays, comme le Zaire, les seules données sur les migrations coloniales sont à tirer des tableaux répartissant la population en population "coutumière" et "extra-coutumière". Ces statistiques auront évidemment fortement sous-estimé l'intensité et la diversité des formes des migrations.

La période post-coloniale se caractérise par un changement assez subtil du discours sur la démographie africaine. En effet, alors que, on l'a vu, l'Afrique coloniale était plutôt considérée comme sous-peuplée, et que le manque de main-d'oeuvre y a été un problème à résoudre pour réussir l'exploitation de l'oeuvre coloniale, l'Afrique post-coloniale sera au contraire taxée de surpeuplée. Une des conséquences de cet état de choses est le grand intérêt porté sur les études de planning familial, la forte croissance démographique étant considérée comme un frein au développement. Quant aux migrations, l'intérêt relativement moindre de les étudier est dû, on l'a vu, au rôle qu'elles jouent dans l'accroissement spectaculaire et problématique des centres urbains ainsi que le déséquilibre dans la répartition spatiale de la population. On comprend alors que les migrations rurales-urbaines aient bien plus attiré l'attention (cfr Simmons, 1985). Les autres formes de migrations (migrations rurales-rurales, urbaines-rurales, circulaires,...) sont donc encore gravement méconnues. Elles ont fait l'objet de bien peu d'opérations de collecte de données.

Plusieurs innovations méthodologiques ont été suggérées pour venir à bout de l'absence relative des données sur les migrations. Il ne nous parait pas opportun de reprendre ici les résultats de ces travaux (cfr Chaire Quételet 1983, ECA/BIS,...). Nous nous limiterons à quelques aspects qui nous paraissent pertinents par rapport à notre problématique, notamment sur la qualité des sources de données et la définition, donc l'observation, du phénomène migratoire. 
La liste de variables à manipuler pour étudier la dialectique migrationdéveloppement suggère qu'il est difficile qu'une seule source puisse offrir toutes les données nécessaires. D'où la nécessité, maintes fois exprimée par plusieurs auteurs (Gregory, 1989 ; Piché, 1989 ; Simmons, 1985), de combiner plusieurs sources : enquêtes ponctuelles, recherche documentaire, recensement, enquêtes post-censitaires, etc. De toutes ces sources, l'enquête semble être la source la plus appropriée pour plusieurs raisons. Deux d'entre elles nous paraissent très importantes : la possibilité de saisir toutes les formes de migrations et celle de saisir à la fois les variables sur la migration et la situation socio-économique (cfr Courgeau, 1988).

L'étude de la dialectique migration-développement exige en effet que toutes les formes de migration soient saisies et analysées. Les migrations temporaires, par exemple, peuvent avoir plus d'impact sur le développement des milieux ruraux que les migrations définitives (cfr Gregory et Piché, 1985 ; Courgeau, 1988). La typologie proposée par Standing (voir annexe I) nous paraît à cet égard très pertinente, car elle propose de définir la migration en considérant, outre les trois critères classiques (espace, résidence et temps), un quatrième, à savoir l'activité économique. Ce qui permet, nous semble-t-il, de saisir une gamme relativement plus complète de migrations vues en rapport avec le processus de développement/sous-développement.

L'enquête offre également la possibilité de saisir à la fois les données sur la migration et la situation socio-économique. C'est notamment le cas des enquêtes rétrospectives des biographies. Une adaptation de cet outil au cas africain a consisté, récemment, à utiliser la fiche "AGEVEN" (Age-évènement) (cfr Antoine, Bry et Diouf, 1987 et Lututala, 1990). Il faut tout de suite dire cependant que tout en étant pertinentes, ces enquêtes constituent encore pour le moment un défi de taille, qu'elles soient menées dans les pays développés (cfr Riandey, 1985) ou sous-développés. Parmi ces limites, on peut mentionner l'appel à la mémoire, la durée de l'interview; la cohérence des biographies et les limites des concepts démographiques utilisés (cfr Riandey, 1985) qui, d'après notre expérience, invitent à une grande prudence lors de la conduite de ces enquêtes en Afrique. D'autres limites doivent aussi être soulevées : la difficulté de recueillir les renseignements sur la biographie auprès d'autres personnes que les migrants eux-mêmes; la difficulté de faire comprendre aux enquêtés, mais aussi aux enquêteurs, que les différents lieux à considérer sont ceux où l'individu aura passé au moins six mois ; l'impossibilité de saisir la dynamique entre l'individu-migrant et les autres membres familiaux, locaux et extra-locaux ; et enfin la difficulté de procéder à la saisie et à l'analyse des renseignements recueillis. Nous reviendrons sur ce dernier aspect plus loin.

Quelques alternatives existent pour contourner les difficultés méthodologiques signalées ci-dessus. On peut citer ici le fait de collecter les histoires migratoires, ou de recueillir les informations socio-économiques pour des courtes périodes ; la conduite d'enquêtes qualitatives diverses, etc.

Sur la définition du phénomène, mentionnons la remise en question du concept classique des migrations dans le cas de l'Afrique, lequel concept ne prend pas en considération une des spécificités du vécu quotidien des 
Africains, à savoir leur ubiquité résidentielle (Lututala, 1989). Une telle conceptualisation rend évidemment les statistiques actuelles sur les migrations et les résultats qu'on en tire inappropriés. Elle exige de nouvelles orientations pour définir et saisir les migrations sur le terrain. Les critères classiques (changement du lieu de naissance ou de résidence et durée d'absence à ce lieu) semblent à la fois étroits et insuffisants pour appréhender l'acte migratoire en rapport avec le développement de l'Afrique.

Par ailleurs, le critère "espace" (changement de lieu de naissance ou de résidence) pose un problème de taille que chercheurs et décideurs politiques doivent résoudre. En effet, s'il est facile d'identifier les migrants "primaires" (voir Lututala, 1987 et 1989), le statut migratoire des descendants (enfants de migrants) fait encore l'objet de débats, notamment dans le cas des migrations internationales. Faut-il considérer ces descendants, dont le lieu de naissance se trouve être le lieu de résidence actuelle ou antérieure des parents, comme des immigrants ? Un travail d'harmonisation des critères scientifiques de définition des migrations et des critères politiques d'attribution de la nationalité ne devrait-il pas être réalisé ? Ce travail doit permettre de répondre à la question de savoir jusque quand une personne qui immigre à un endroit doit-elle y être considérée comme "migrante". Sur le plan strictement démographique, la question est de trouver la durée maximale qu'il faut considérer, à côté de la durée minimale ( 3 ou 6 mois), pour qu'une personne cesse d'être migrante.

L'harmonisation des critères de définition des migrations s'impose également entre démographes et spécialistes d'autres disciplines. On constate en effet que les critères utilisés par les démographes pour mieux observer les migrations ne sont pas toujours ceux qu'utilisent les sociologues, économistes, et autres planificateurs. Il n'est pas rare que ceux-ci définissent la migration comme un simple dépassement des frontières, sans se reférer aux critères temps et changement d'espace.

Un autre défi qu'il convient de relever concerne la saisie des migrations internationales à partir de la question sur la nationalité ou le pays d'origine que l'on retrouve dans certaines sources (registres des frontières, par exemple). Cette question est de plus en plus inappropriée notamment dans les régions frontalières où les immigrants ont un problème d'identité et ont tendance à décliner plutôt la nationalité du pays hôte, tout en gardant celle du pays d'origine. La confrontation des réponses fournies par le chef de ménage et la vérification de ses pièces d'identité au moment des interviews est loin de résoudre le problème tant la disposition frauduleuse des pièces d'identité du pays hôte est monnaie courante. Le recours aux caractéristiques morphologiques (taille, figure,...) ne peut tenir que pour certains peuples et ethnies et conduirait dans tous les cas à de graves erreurs d'appréciation et de sous ou sur-estimation du nombre de migrants.

\subsection{L'absence des méthodes d'analyse}

L'étude des migrations souffre aussi d'une absence ou de la rigueur des méthodes d'analyse. En démographie, cette lacune trouverait ses origines 
historiques à la fois dans le moindre intérêt sur le phénomène et à la faible intensité qu'il aurait accusé au moment où naissait et se développait cette discipline. C'est ainsi que la mortalité et la fécondité ont attiré plus d'attention et fait l'objet de plus de préoccupations méthodologiques. Ainsi s'expliquerait le fait que des manuels entiers de démographie ne comportent aucun chapitre sur la migration (cfr ancien manuel de Pressat) ou réservent au phénomène sept.(!) pages alors que la mortalité, par exemple, en occupe 125 (cas du manuel de Yaoundé).

Pour combler cette lacune, certaines méthodes ont été forgées, notamment pour analyser les données sur les biographies (cfr Courgeau, 1989). La rigueur de ces techniques est telle qu'elles restent peu utilisées pour l'étude des migrations africaines. A cette rigueur, il faut aussi ajouter le fait qu'il faut disposer de l'infrastructure nécessaire pour appliquer ces méthodes : logiciels appropriés, équipement informatique, etc. Ceci conduit donc souvent à abandonner l'exploitation et l'analyse des données sur les biographies, et à des gaspillages de fonds engloutis dans la collecte. La question est importante tant les analyses qui sont faites des enquêtes sur les migrations contrastent très largement avec la richesse des informations qui sont contenues dans les questionnaires.

Les méthodes d'analyse des biographies se concentrent essentiellement sur les individus-migrants et ne peuvent par conséquent rendre compte de la dialectique migration-développement au niveau macro et méso. Le recours à l'une ou l'autre des techniques d'analyse multivariée est donc essentiellement dans ce cas. L'étude des déterminants de la migration peut être réalisée en ayant recours à la régression multiple, tandis que celle de l'identification des "patterns" peut être faite en utilisant l'analyse factorielle.

On doit mentionner enfin la difficulté de coupler les informations macro, méso et micro recueillies de différentes sources. Bien qu'il ne soit pas impossible, ce couplage nécessite des logiciels assez sophistiqués, ce qui en compromet l'utilisation dans l'état actuel de l'infrastructure informatique africaine.

\section{Conclusion}

Il est généralement admis que les migrations sont intimement liées au processus de développement des pays africains. D'où l'impérieuse nécessité de prendre en compte ce phénomène dans la planification du développement de l'Afrique. Seulement, la réalisation de ce souhait est handicapée par des écueils méthodologiques. Nos connaissances sur les migrations africaines ressemblent à un tonneau vide et un iceberg. Il s'agirait d'un tonneau vide qui résonne fort tandis qu'il ne contient rien. Que sait-on des causes et conséquences de la migration ? Comment expliquer cette vision positiviste des économistes néo-classiques vis-à-vis du phénomène pendant qu'il est perçu tantôt comme favorisant, tantôt comme bloquant le processus de développement ? 
Si les connaissances sur les migrations sont encore à affiner, c'est parce qu'il se pose encore des problèmes méthodologiques. Nous avons examiné, dans ce papier le problème de l'absence des données, de la mauvaise conceptualisation de la dialectique migration-développement et du concept migration lui-même, de l'absence ou la rigueur des méthodes d'analyse. Ces questions doivent être réglées au préalable, pour permettre une meilleure appréhension du rôle et de la place de la migration dans le processus de développement de l'Afrique. 


\section{BIBLIOGRAPHIE}

ANTOINE, Ph.; BRY, X., et Demba DIOUF

BIISBORROW, R.E.

1981

BILSBORROW, R.E.

COURGEAU, D.

1988

COURGEAU, D. et EVa LELIEVRE

FAROOQ, G. and PERNIA, E.M.
1987

$$
\begin{aligned}
& \text { "La fiche 'AGEVEN': un outil } \\
& \text { pour la collecte des données } \\
& \text { rétrospectives", Techniques } \\
& \text { d'enquête. vol } 13, \mathrm{n}^{\circ} 2 \text {, } \\
& \text { Pp. 173-181 }
\end{aligned}
$$
"Priority areas for future research on demographic- economic interrelationships", in United Nations,
Population and Development Modeling. Population Studies, $n^{\circ} 73$, New York, pp. 74-87

Guide pour des enquêtes sur les migrations internes dans les pays en voie de développement: des variables communautaires, BIT, Genève, $83 \mathrm{p}$.

Méthodes de mesure sur la mobilité spatiale. Paris, I.N.E.D., $307 \mathrm{p}$.

Analyse démographique des biographies. Paris, I.N.E.D., 208 p.
"Need for and approaches to in- tegrated population, human resource and development plan- ning", Population Bulletin of the United Nations. Nos 23/24, pp. $11-20$


GREGORY, J.W.

1989

GREGORY, J.W. et PICHE, V.

LOCOH, T.

LUTUTALA, M.

1987

LUTUTALA, M.

1989

LUTUTALA, M.

1990
"L'insertion des migrantes et migrants en ville - Interrogations méthodologiques", in ANTOINE, Ph. et COULIBALY, S. (éds), L'insertion urbaine

des migrants en Afrique, Paris, ORSTOM, 1989, pp. 161-172

"La migration africaine vue à travers le prisme des enquêtes récentes", in Chaire Quételet '83 : Migrations internes Collecte des données ét méthodes d'analyse, Louvain-1a-Neuve, Cabay, pp. 305-357

"La migration des enfants rapportés par les mères - Application au Sud-Togo", dans Chaire Ouételet '83 : Migrations in ternes - Collecte des données et méthodes d'analyse, Louvainla-Neuve, Cabay, pp. 377-390

Dynamique des migrations au Zaire - le réseau de Kinshasa, Montréal, Thèse de doctorat, Université de Montréal, 424 p.

"L'ubiquité résidentielle africaine et le concept des migrations", Etude sur 1a Population africaine, $n^{\circ} 2$, pp. 5-17

Les villes secondaires dans les processus migratoires au Zaïre, CODESRIA: PPS-PPD, Rapport de recherche, (en préparation) 
NATIONS UNIES

1985

PICHE, V.

1989

RIANDEY, B.

SIMMONS, A.B.

1982

SIMMONS, A.B.

1985
Répartition de la population, migration et développement: grandes questions des années ' 80 ", Bulletin démographique des Nations Unies, $n^{\circ} 16$, pp. 30-46

"L'immigraiton haitienne au Québec - Modalités d'insertion", in ANTOINE, Ph. et COULIBALY, S. (éds), L'insertion urbaine des migrants en Afrique, Paris, ORSTOM, 1989, pp. 201-221

"L'enquête 'Biographie familiale, professionnelle et migratoire' (INED, 1981) - Le bilan de la collecte", dans Chaire Quételet '83: Migratioins internes - Collecte des données et méthodes d'analyse. Louvain-la-Neuve, Cabay, pp. 117133

"Hypotheses and analytic approaches for the study of the demographic and socio-economic consequences of migration", U.N./ ESCAP, National Migration Surveys $X$. Guidelines for Analyses, New York, 163-188

"Methodological Innovations for Survey Studies of Third World Migration", in Chaire Quételet '83 : Migrations internes - Collecte des données et méthodes d'analyse, Louvain-la-Neuve, Cabay, pp. 205-241 
http://aps.journals.ac.za

STANDING, G.

1983

"Conceptualising Territorial

Mobility" in Bilsborrow, R.E.,

A.S. Oberai andG. Standing (eds)

Migration Surveys in Low-Income

Countries, London and Sydney,

BIT/Croom Helm, pp. 31-59 


\section{ANNEXE I}

\section{Typologie migratoire de STANDING,-G.}

1. Migrants permanents ( changent le lieu d'activité économique mais pas nécessairement celui de résidence)

1.1. Nomades

1.1.2. Ramasseurs

1.1.3. Vagabonds

1.2. Travailleurs migrants

2. Migrants temporaires (= changent l'activité économique mais non le lieu de résidence habituel)

2.1. Migrants circulaires ou pendulaires ou rotatifs ou à "court terme"

2.2.1. Migrants saisonniers

2.2.2. Migrants "compensatoires"

2.2. Migrants pour une période donnée de la vie

2.3. "Commuters"

3. Migrants transférés (= changent de lieu de résidence et celui de l'activité économique)

4. Migrants à long terme (= changent de lieu de résidence et celui de l'activité économique)

4.1. Migrants-durée de vie active

4.2, Migrants à vie

4.1.1./4.2.1. Migrants primaires

4.1.2./4.2.2. Migrants répétitifs 
4.3. Migrants de retour

4.4. Migrants par étapes

4.5. Migrants circulaires

5. Non-migrants

5.1. Résidents à vie

5.2. Résidents indécis

5.3. Migrants potentiels

source : Standing (1984) 